\title{
Average-Case Performance Evaluation of Online Algorithms for Routing and Wavelength Assignment in WDM Optical Networks
}

\author{
Keqin Li \\ Department of Computer Science \\ State University of New York \\ New Paltz, New York 12561, USA \\ lik@newpaltz.edu
}

\begin{abstract}
We investigate the problem of online routing and wavelength assignment and the related throughput maximization problem in wavelength division multiplexing optical networks. It is pointed out that these problems are highly inapproximable. We evaluate the average-case performance of several online algorithms, which have no knowledge of future arriving connection requests when processing the current connection request. Our experimental results on a wide range of optical networks demonstrate that the averagecase performance of these algorithms are very close to optimal.
\end{abstract}

\section{Introduction}

Given wavelengths $\lambda_{1}, \lambda_{2}, \lambda_{3}, \ldots$, and a sequence of connection requests $\sigma=\left(r_{1}, r_{2}, \ldots, r_{m}\right)$ in a wavelength division multiplexing (WDM) network, where each connection request $r_{j}$ is a source-destination pair $r_{j}=\left(s_{j}, d_{j}\right)$, $1 \leq j \leq m$, the routing and wavelength assignment (RWA) problem is to establish a lightpath $p_{j}$ for each connection request $r_{j}$ and assign a wavelength $\lambda_{i_{j}}$ to each lightpath $p_{j}$, where $1 \leq i_{j} \leq k$, such that no two lightpaths which share a common link are assigned the same wavelength and that the number $k$ of wavelengths used is minimized. We also consider a related optimization problem of RWA, namely, the throughput maximization (TM) problem, in which we are given a fixed number $k$ of wavelengths $\lambda_{1}, \lambda_{2}, \ldots, \lambda_{k}$, and a sequence $\sigma$ of connection requests. The goal is to satisfy as many connection requests as possible by using the $k$ wavelengths.

Both the RWA and the TM problems contain two subproblems, namely, routing (finding a lightpath for each connection request) and coloring (assigning a wavelength to

1-4244-0910-1/07/\$20.00 (c)2007 IEEE. each lightpath). Each subproblem alone makes the RWA and TM problems NP-hard. When a lightpath is given for each connection request, the RWA problem becomes the wavelength assignment (WA) problem. It has been proven that the WA problem and the well known NP-hard graph coloring problem can be reduced to each other [12]. Hence, the WA problem has high inapproximability; in particular, if $\mathrm{NP} \neq \mathrm{ZPP}$, for any constant $\delta>0$, no polynomial time WA algorithm can achieve approximation ratio $n^{1 / 2-\delta}$ or $m^{1-\delta}$ for $m$ lightpaths in an $n$-node WDM network [23]. When there is only one wavelength, the TM problem is precisely the classical maximum disjoint paths (MDP) problem, that is, finding as many edge-disjoint paths as possible for a sequence $\sigma$ of source-destination pairs. The MDP problem is also highly inapproximable; in particular, if $\mathrm{P} \neq \mathrm{NP}$, for any constant $\delta>0$, no polynomial time MDP algorithm can achieve approximation ratio $m^{1 / 2-\delta}$ for a WDM network with $m$ edges [14].

The RWA and TM problems have been extensively studied by many researchers in the last ten years. Various heuristic methods have been proposed, such as genetic algorithms [9], graph-theoretic modeling [11], partition coloring [22], integer linear program [5, 27]. A recent survey of various algorithms for the RWA problem can be found in [13]. The reader is also referred to [28] for information on WDM optical networks.

In this paper, we consider online routing and wavelength assignment in WDM optical networks, where connection requests arrive in the order of $\sigma$, one at a time. Upon the arrival of a connection request $r_{j}$, a lightpath $p_{j}$ is established and its wavelength is assigned immediately without knowing the remaining connection requests $r_{j+1}, r_{j+2}, \ldots, r_{m}$, but only the past connection requests $r_{1}, r_{2}, \ldots, r_{j-1}$. Online RWA and TM algorithms are very useful in real applications, since connection requests typically do not arrive at the same time, and those arriving earlier should be processed before the entire sequence of requests is available. 
It is not surprising that the online RWA and TM problems are highly inapproximable, since the offline RWA and TM problems already contain highly inapproximable graph coloring and disjoint paths problems as subproblems or special cases. Nevertheless, it is still possible that there exist effective approximation algorithms with excellent average-case performance. The main contribution of the paper is to develop several online RWA and TM algorithms and demonstrate by experimentation that the average-case competitive ratios of these algorithms are very close to optimal. It should be noticed that while existing work only compare heuristic algorithms with themselves, we are able to compare the performance of our algorithms with optimal solutions (actually, lower bounds for the optimal solutions).

\section{Inapproximability of Online RWA and TM Problems}

Let $\operatorname{ALG}(\sigma)$ denote the solution produced by algorithm $\operatorname{ALG}$ and $\operatorname{OPT}(\sigma)$ the optimal solution for an instance $\sigma$. For example, in the RWA problem, $\operatorname{ALG}(\sigma)$ denotes the number of wavelengths needed by algorithm ALG to establish lightpaths for the connection requests in $\sigma$, and $\mathrm{OPT}(\sigma)$ denotes the minimum number of wavelengths needed to support the connection requests in $\sigma$. In the TM problem, $\operatorname{ALG}(\sigma)$ denotes the number of lightpaths established by algorithm ALG for the connection requests in $\sigma$ by using the given number of wavelengths, and $\operatorname{OPT}(\sigma)$ denotes the maximum number of lightpaths that can be established for the connection requests in $\sigma$. The competitive ratio of an online algorithm ALG is defined as

$$
\sup _{\sigma}\left(\frac{\operatorname{ALG}(\sigma)}{\operatorname{OPT}(\sigma)}\right), \quad \text { for a minimization problem; }
$$

and

$$
\sup _{\sigma}\left(\frac{\operatorname{OPT}(\sigma)}{\operatorname{ALG}(\sigma)}\right), \quad \text { for a maximization problem. }
$$

Algorithm ALG is said to be $\alpha$-competitive, if for all $\sigma$,

$$
\operatorname{ALG}(\sigma) \leq \alpha \cdot \operatorname{OPT}(\sigma), \quad \text { for a minimization problem; }
$$

and

$$
\operatorname{ALG}(\sigma) \geq \frac{1}{\alpha} \cdot \operatorname{OPT}(\sigma), \quad \text { for a maximization problem. }
$$

For a randomized algorithm, $\operatorname{ALG}(\sigma)$ is replaced by $E(\operatorname{ALG}(\sigma))$, where $E(\cdot)$ denotes the expectation of a random variable [10].

The RWA problem is also called path coloring (PC) problem. Online path coloring has been studied extensively in the literature. It was shown that there is a 3 competitive algorithm (called Recursive Greedy) for path coloring on linear array networks and no deterministic online algorithm is better than 3-competitive [19]. For any $n$-node tree network, it was shown that both the Classifyand-Greedy-Color algorithm [8] and the First-Fit-Coloring [18] algorithm are $2 \log n$-competitive. It was also proven in [8] that any deterministic algorithm has competitive ratio at least $\Omega\left(\frac{\log n}{\log \log n}\right)$ even for complete binary tree networks. Bartal and Leonardi also constructed the optimal $O(\log n)$-competitive algorithm for path coloring on $n \times n$ mesh networks. On brick wall graphs, it was shown that any randomized algorithm is at best $n^{1-\log _{4} 3}$-competitive [7], where $1-\log _{4} 3=0.2075187 \ldots$.

The lower bound for brick wall graphs implies that no deterministic or randomized online routing and wavelength assignment algorithm has reasonable competitiveness, especially for large networks. The above discussion gives rise to the following inapproximability theorem for the routing and wavelength assignment problem on arbitrary networks.

Inapproximability Theorem 1. For n-node WDM optical networks, there is no deterministic or randomized online routing and wavelength assignment algorithm that has a competitive ratio less than $n^{0.2075}$.

When there is only one wavelength, the TM problem becomes the MDP problem. It is a simple observation that any deterministic online algorithm for the MDP problem has competitive ratio at least $n-1$ even on an $n$-node linear array network [2]. Therefore, investigation has been focused on randomized algorithms. Lower bounds for randomized algorithms for the MDP problem on linear array networks were established in [3]. For tree networks with diameter $D$, several $O(\log D)$-competitive algorithms have been developed $[3,4,21]$. The lower bound $\Omega(\log n)$ and the optimal $O(\log n)$ upper bound for randomized algorithms on $n \times n$ mesh networks are found in [4] and [20] respectively. The randomized lower bound of $n^{0.2075}$ for brick wall graphs is due to [7].

The lower bound for brick wall graphs implies the following inapproximability theorem for the throughput maximization problem on arbitrary networks.

Inapproximability Theorem 2. For n-node WDM optical networks, there is no deterministic or randomized online throughput maximization algorithm that has a competitive ratio less than $n^{0.2075}$.

\section{Lower Bounds}

The solutions produced by an approximation algorithm should be compared with optimal solutions. Unfortunately, it is infeasible to obtain optimal routing and wavelength assignment in reasonable amount of time even for moderate 
sized networks. In this section, we derive lower bounds for the minimum number of wavelengths required.

A cutset $C$ of a connected graph (WDM network) is a set of $W(C)$ edges (optical links) $C=\left\{l_{1}, l_{2}, \ldots, l_{W(C)}\right\}$ whose removal results in disconnection of the network [17], i.e., a partition of the network into two subnetworks with $n(C)$ and $n-n(C)$ nodes respectively. For a sequence $\sigma=\left(r_{1}, r_{2}, \ldots, r_{m}\right)$ of connection requests, let $m(\sigma, C)$ denote the number of connection requests $r_{j}=\left(s_{j}, d_{j}\right)$ in $\sigma$ such that $s_{j}$ and $d_{j}$ are in the two disjoint subnetworks separated by the cutset $C$. For each such $r_{j}$, the lightpath established for $r_{j}$ must go through one of the $W(C)$ links $l_{1}, l_{2}, \ldots, l_{W(C)}$. Let $L_{l}$ be the load on an optical link $l$, i.e., the number of lightpaths passing through $l$. Then, the maximum load on $l_{1}, l_{2}, \ldots, l_{W(C)}$ is at least

$$
\max _{1 \leq i \leq W(C)}\left(L_{l_{i}}\right) \geq \frac{m(\sigma, C)}{W(C)} .
$$

Since

$$
\operatorname{OPT}(\sigma) \geq \max _{1 \leq i \leq W(C)}\left(L_{l_{i}}\right)
$$

we obtain

$$
\operatorname{OPT}(\sigma) \geq \frac{m(\sigma, C)}{W(C)} .
$$

The above lower bound is strengthened to

$$
\operatorname{OPT}(\sigma) \geq \max _{C}\left(\frac{m(\sigma, C)}{W(C)}\right),
$$

because $C$ can be an arbitrary cutset.

The minimum size $W$ of a cutset that results in an even partition of a network into two subnetworks of sizes $\lfloor n / 2\rfloor$ and $\lceil n / 2\rceil$ is called the bisection width of the network. By considering a cutset $C$ with $W$ links, we get a special lower bound for $\operatorname{OPT}(\sigma)$ :

$$
\operatorname{OPT}(\sigma) \geq \frac{m(\sigma, C)}{W} .
$$

The above discussion is summarized as the following theorem.

Lower Bound Theorem A. For any WDM network and a sequence $\sigma$ of connection requests, we have

$$
\operatorname{OPT}(\sigma) \geq \max _{C}\left(\frac{m(\sigma, C)}{W(C)}\right) .
$$

In particular, for a cutset $C$ with $W(C)$ equal to the network's bisection width $W$, we have

$$
\operatorname{OPT}(\sigma) \geq \frac{m(\sigma, C)}{W} .
$$

(Note: The above lower bound is valid for both online and offline RWA problems.)
Now we derive a lower bound for $E(\operatorname{OPT}(\sigma))$, where $\sigma$ is a sequence of $m$ random connection requests $r_{1}, r_{2}, \ldots, r_{m}$. We consider two models of random connection requests. In the random drawing with replacement model, each connection request $r_{j}=\left(s_{j}, d_{j}\right)$ is a source-destination pair drawn from the set of $n(n-1) / 2$ possible pairs randomly with a uniform distribution. For such a randomly chosen connection request $r_{j}=\left(s_{j}, d_{j}\right)$, the probability that $s_{j}$ and $d_{j}$ are in the two separate parts of the network is

$$
\frac{n(C)(n-n(C))}{n(n-1) / 2} .
$$

Hence, for $m$ independent random connection requests, the expected number of lightpaths passing through $l_{1}, l_{2}, \ldots, l_{W(C)}$ is

$$
E(m(\sigma, C))=\frac{n(C)(n-n(C))}{n(n-1) / 2} \cdot m .
$$

In the random drawing without replacement model, the sequence $\sigma$ contains $m$ distinct connection requests $r_{1}, r_{2}, \ldots, r_{m}$. Therefore, the number $m(\sigma, C)$ of connection requests $r_{j}=\left(s_{j}, d_{j}\right)$ with $s_{j}$ and $d_{j}$ in the two separate parts of the network is a hypergeometric random variable, i.e.,

$$
\begin{gathered}
P\{m(\sigma, C)=i\}= \\
\frac{\left(\begin{array}{c}
n(C)(n-n(C)) \\
i
\end{array}\right)\left(\begin{array}{c}
n(n-1) / 2-n(C)(n-n(C)) \\
m-i
\end{array}\right)}{\left(\begin{array}{c}
n(n-1) / 2 \\
m
\end{array}\right)},
\end{gathered}
$$

for all $0 \leq i \leq m[15]$. The expectation of $m(\sigma, C)$ is

$$
E(m(\sigma, C))=\frac{n(C)(n-n(C)) m}{n(n-1) / 2} .
$$

In both models, the maximum expected number of lightpaths passing through one of $l_{1}, l_{2}, \ldots, l_{W(C)}$ is at least

$\max _{1 \leq i \leq W(C)}\left(E\left(L_{l_{i}}\right)\right) \geq \frac{E(m(\sigma, C))}{W(C)}=\frac{n(C)(n-n(C))}{n(n-1) / 2} \cdot \frac{m}{W(C)}$.

Since

$$
E(\mathrm{OPT}(\sigma)) \geq E(L) \geq \max _{1 \leq i \leq W(C)}\left(E\left(L_{l_{i}}\right)\right),
$$

we have the following lower bound for $E(\mathrm{OPT}(\sigma))$ :

$$
E(\operatorname{OPT}(\sigma)) \geq \frac{n(C)(n-n(C))}{n(n-1) / 2} \cdot \frac{m}{W(C)} .
$$

The above lower bound is strengthened to

$$
E(\operatorname{OPT}(\sigma)) \geq \max _{C}\left(\frac{n(C)(n-n(C))}{n(n-1) / 2} \cdot \frac{m}{W(C)}\right),
$$


because $C$ can be an arbitrary cutset. By considering a cutset $C$ with $W(C)$ equal to the bisection width $W$, we get a special lower bound for $E(\mathrm{OPT}(\sigma))$ :

$$
E(\mathrm{OPT}(\sigma)) \geq \frac{\lfloor n / 2\rfloor\lceil n / 2\rceil}{n(n-1) / 2} \cdot \frac{m}{W} .
$$

The above discussion is summarized as the following theorem.

Lower Bound Theorem B. For any n-node WDM network and a sequence $\sigma$ of $m$ random connection requests, we have

$$
E(\mathrm{OPT}(\sigma)) \geq \max _{C}\left(\frac{n(C)(n-n(C))}{W(C)}\right) \cdot \frac{m}{n(n-1) / 2} .
$$

In particular, if the network has bisection width $W$, we have

$$
E(\operatorname{OPT}(\sigma)) \geq \frac{\lfloor n / 2\rfloor\lceil n / 2\rceil}{n(n-1) / 2} \cdot \frac{m}{W} \approx \frac{m}{2 W} .
$$

(Note: The above lower bound is valid for both online and offline RWA problems.)

Both Lower Bound Theorems A and B are applicable to the random drawing with/without replacement models.

\section{Online Algorithms}

While the known results on the worst-case performance of online PC and MDP problems are quite discouraging (i.e. the RWA and the TM problems have high inapproximability for arbitrary WDM networks), we take a different approach to attacking the online RWA and TM problems in this paper, that is, evaluating the average-case performance of (deterministic and randomized) online algorithms.

Let $\sigma$ denote a sequence of $m$ random connection requests $r_{1}, r_{2}, \ldots, r_{m}$. For such random input, both ALG $(\sigma)$ and $\operatorname{OPT}(\sigma)$ become random variables. We also notice that ALG can be a randomized algorithm and a WDM network can be a random network. We define two average-case competitive ratios

$$
\alpha(\mathrm{ALG})=E\left(\frac{\operatorname{ALG}(\sigma)}{\operatorname{OPT}(\sigma)}\right),
$$

and

$$
\beta(\mathrm{ALG})=\frac{E(\mathrm{ALG}(\sigma))}{E(\mathrm{OPT}(\sigma))},
$$

where the expectations are taken over

- all sequences of $m$ random connection requests;

- all random choices of algorithm ALG if it is a randomized algorithm;
- all samples of a random network.

The above three sources of randomness are independent of each other.

We will evaluate the average-case performance of several online algorithms for the RWA and the TM problems. All our algorithms visualize a WDM optical network $N=(V, E)$ as having separate copies, $N_{1}, N_{2}, N_{3}, \ldots$, one for each wavelength, such that all the connection requests routed on $N_{i}$ use the wavelength $\lambda_{i}$, and that lightpaths on the same copy $N_{i}$ are edge-disjoint. Initially, there is only one copy $N_{1}$, and new copies will be introduced when necessary.

Assume that $N_{1}, N_{2}, \ldots, N_{b}$ are the current copies ever used. When processing a connection request $r_{j}$, an existing copy $N_{i}$ is chosen to find a lightpath $p_{j}$ for $r_{j}$ and the lightpath $p_{j}$ is assigned the wavelength $\lambda_{i}$. Then, the optical links occupied by $p_{j}$ are deleted from $N_{i}$, so that these links cannot be used by later connection requests to prevent link overlapping.

Different algorithms use different strategies in identifying $N_{i}$. We will consider the following heuristics.

- First-Fit (FF) - A shortest lightpath is sought in $N_{1}$ by using those optical links still not deleted. If there is no such a lightpath, a shortest lightpath is sought in $N_{2}$, $N_{3}, \ldots$, and so on, until a lightpath is found.

- Best-Fit (BF) - A shortest lightpath $p_{j, i}$ is sought in each of $N_{i}, 1 \leq i \leq b$. Then, the shortest lightpath among $p_{j, 1}, p_{j, 2}, \ldots, p_{j, b}$ is chosen as $p_{j}$.

- Densest-Fit (DF) - A shortest lightpath is sought in $N_{i}$ which has the most optical links among $N_{1}, N_{2}, \ldots, N_{b}$. If such a lightpath cannot be established, a shortest lightpath is sought in the copy with the second most links, the copy with the third most links, ..., and so on, until a lightpath is found.

- Random-Fit (RF) - A shortest lightpath is sought in a randomly selected copy $N_{i}$, where $N_{i}$ is chosen from all those copies which can provide shortest paths for $r_{j}$, say, $N_{i_{1}}, N_{i_{2}}, N_{i_{3}}, \ldots$, and each of these copies $N_{i_{1}}, N_{i_{2}}, N_{i_{3}}, \ldots$ are chosen with equal probability.

In all the above algorithms, a shortest lightpath is found by using the breadth-first search algorithm.

When no existing copy in $N_{1}, N_{2}, \ldots, N_{b}$ can provide a lightpath for $r_{j}$, a new copy $N_{b+1}$ identical to $N$ is initiated, so that a shortest lightpath $p_{j}$ is established on $N_{b+1}$ and assigned the wavelength $\lambda_{b+1}$. However, for the TM problem, the connection request is blocked (i.e., not satisfied and rejected) if $b$ is already equal to $k$, the given number of wavelengths. 


\section{Experimental Performance Evaluation}

Extensive experiments have been conducted to evaluate the average-case performance of the online algorithms presented in the last section for the RWA and the TM problems on a wide range of WDM optical networks.

\subsection{The Methodology}

In the experiments for the RWA problem, for each combination of (network, algorithm, $m$ ), we report $\bar{\alpha}, \bar{\beta}$, and $\overline{p l}$, whose meanings are explained as follows.

- The lower bound for $\operatorname{OPT}(\sigma)$ expressed in Eq. (1) requires coverage of all cutsets $C$, which is certainly computationally infeasible. Hence, for each network $N$, there are $\eta(N)$ pre-chosen cutsets $C_{1}, C_{2}, \ldots, C_{\eta(N)}$, such that the lower bound for $\operatorname{OPT}(\sigma)$ in Eq. (1) is simplified as

$$
\tilde{l b}=\max _{1 \leq i \leq \eta(N)}\left(\frac{m\left(\sigma, C_{i}\right)}{W\left(C_{i}\right)}\right) .
$$

The above lower bound $\tilde{l b}$ is then used to be compared with $\operatorname{ALG}(\sigma)$. Thus, the following expectation

$$
\bar{\alpha}=E\left(\frac{\operatorname{ALG}(\sigma)}{\tilde{l b}}\right)
$$

is an over-estimation of $\alpha$ (ALG).

- The lower bound for $E(\mathrm{OPT}(\sigma))$ expressed in Eq. (2) also requires coverage of all cutsets $C$. For a particular network $N$, we can always choose a cutset $C_{1}$ which maximizes

$$
\frac{n(C)(n-n(C))}{W(C)} \text {. }
$$

Hence, the lower bound for $E(\mathrm{OPT}(\sigma))$ in Eq. (2) is simplified as

$$
l b=\frac{n\left(C_{1}\right)\left(n-n\left(C_{1}\right)\right)}{W\left(C_{1}\right)} \cdot \frac{m}{n(n-1) / 2} .
$$

However, the following ratio

$$
\bar{\beta}=\frac{E(\operatorname{ALG}(\sigma))}{\frac{n\left(C_{1}\right)\left(n-n\left(C_{1}\right)\right)}{W\left(C_{1}\right)} \cdot \frac{m}{n(n-1) / 2}}
$$

is still an over-estimation of $\beta(\mathrm{ALG})$. For a random network, the lower bound for $E(\mathrm{OPT}(\sigma))$ in Eq. (2) is modified as

$$
\overline{l b}=E\left(\frac{n\left(C_{1}\right)\left(n-n\left(C_{1}\right)\right)}{W\left(C_{1}\right)} \cdot \frac{m}{n(n-1) / 2}\right),
$$

where $C_{1}$ is the random cutset which cuts the unit square into upper and lower halves, and

$$
\bar{\beta}=\frac{E(\operatorname{ALG}(\sigma))}{\overline{l b}} .
$$

(See Section 5.2 for random network generation.)

- In addition to the number of wavelengths to be minimized, the average length $\overline{p l}$ of lightpaths should also be minimized, though this is a secondary optimization goal.

In the experiments for the TM problem, for each combination of (network, algorithm, $m, k$ ), we report $\bar{B}$, which is ( 1 - the expected blocking rate), i.e., the expected percentage of connection requests that are satisfied by using $k$ wavelengths.

\subsection{Optical Networks}

Eight WDM optical networks are considered in our experiments, namely, a mesh network, four real networks, and three types of random networks:

- the $10 \times 10$ mesh network with $\eta=2$ and $C_{1}, C_{2}$ shown in Figure 1;

- a 24-node ARPANET-like regional network [29] with $\eta=5$ and $C_{1}, \ldots, C_{5}$ shown in Figure 2;

- a 16-node NSFNET backbone [6] with $\eta=2$ and $C_{1}, C_{2}$ shown in Figure 3;

- the 20-node European Optical Network (EON) [25] with $\eta=6$ and $C_{1}, \ldots, C_{6}$ shown in Figure 4;

- the 30-node UK Network [1] with $\eta=6$ and $C_{1}, \ldots, C_{6}$ shown in Figure 5;

- 100-node random grid networks;

- 50-node random regular networks;

- 50-node random unit disk networks.

In Figures 1-5, the cutsets are arranged in decreasing order of

$$
\frac{n\left(C_{i}\right)\left(n-n\left(C_{i}\right)\right)}{W\left(C_{i}\right)},
$$

whose values are shown in the parentheses. The cutsets for random networks are described below.

Although a number of models are available in random graph theory, e.g., models A, B, and C in [26], none of them is appropriate to model computer networks. We believe that a random network model should incorporate link locality into consideration. In this research, we consider three types of random networks. 


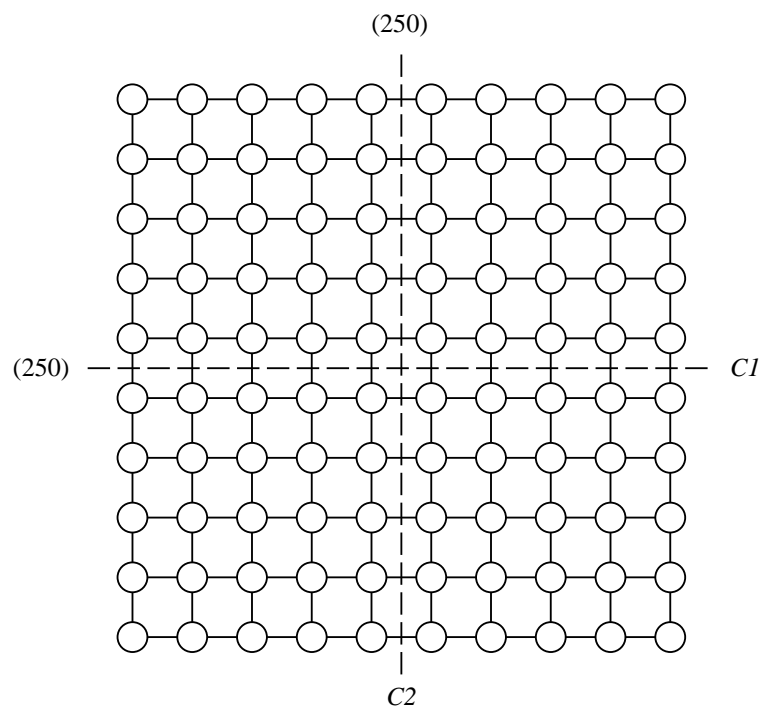

Figure 1. A $10 \times 10$ mesh network.

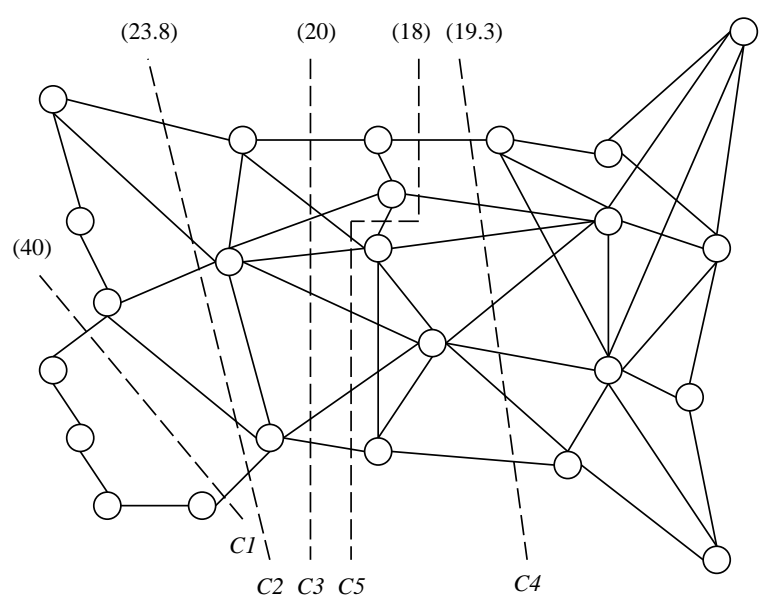

Figure 2. A 24-node ARPANET-like network.

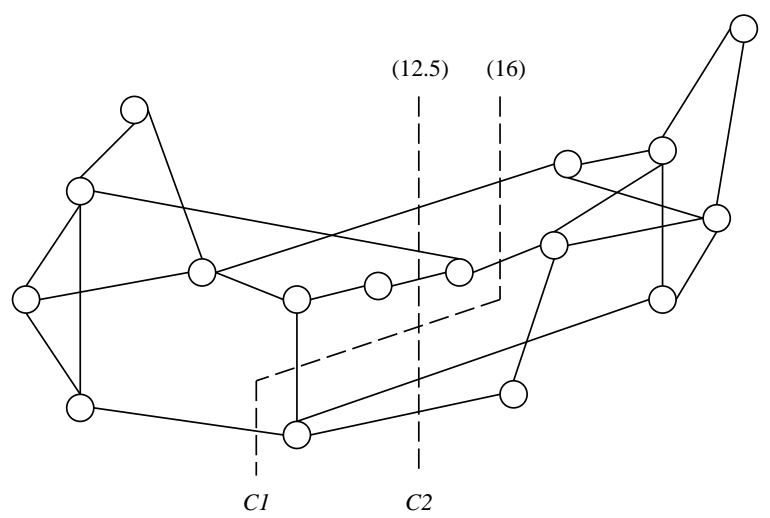

Figure 3. A 16-node NSFNET backbone.

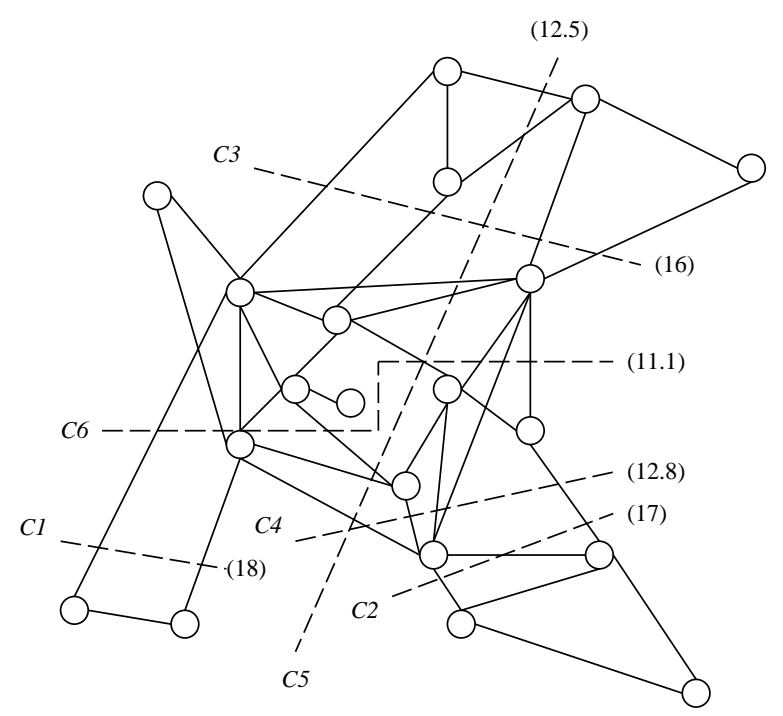

Figure 4. The 20-node European Optical Network.

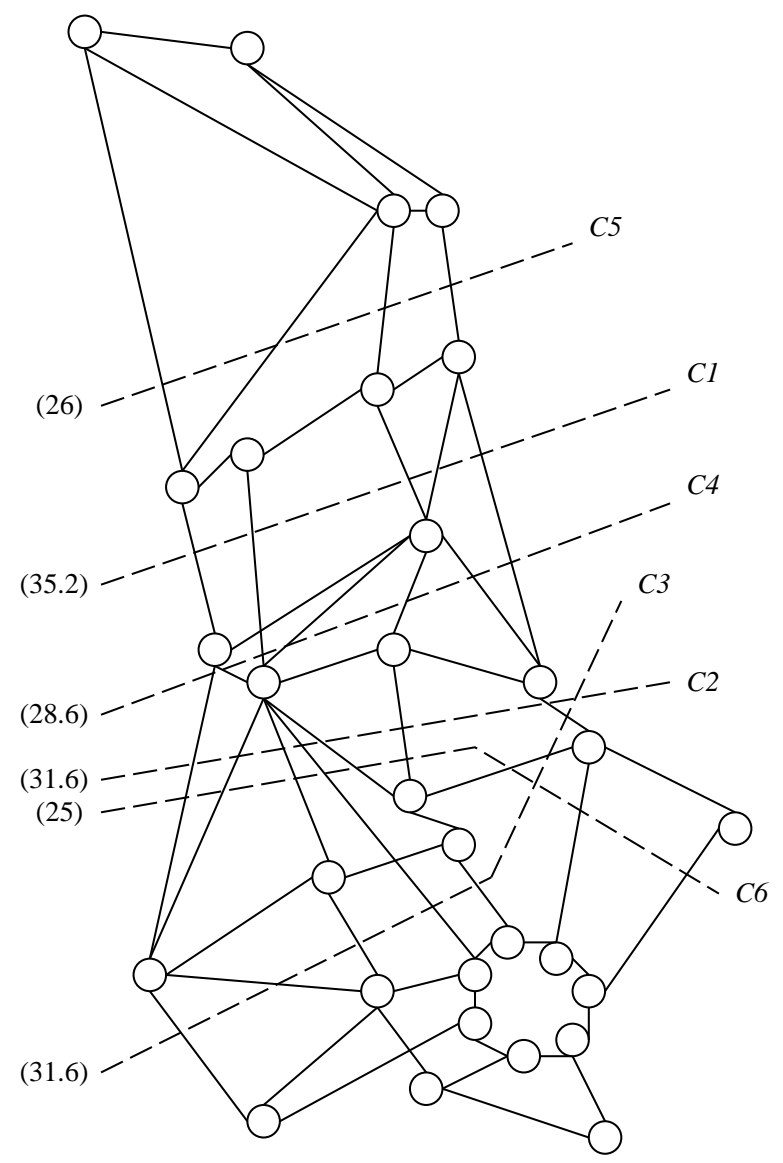

Figure 5. The 30-node UK Network. 

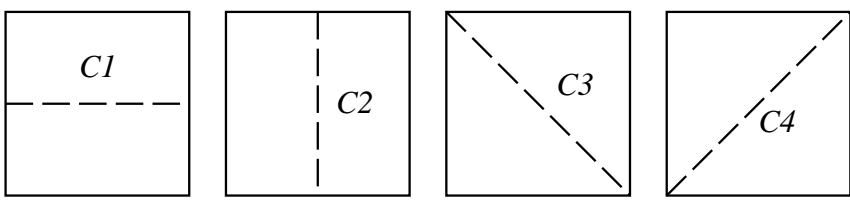

Figure 6. Cutsets in a random network.

A random grid network $N_{q}=(V, E)$ is a subnetwork of the mesh network and is generated as follows. In a $\sqrt{n} \times \sqrt{n}$ grid network, the $n$ nodes in $V$ are identical to the nodes in a $\sqrt{n} \times \sqrt{n}$ mesh network. Each link of the mesh network appears in a random grid network with probability $q$ and is independent of the existence of other links, where $0<q<$ 1. Cutsets for random grid networks are the same as those for mesh networks.

A random regular network $N_{d}=(V, E)$ is generated as follows. Let $U$ be a unit square in the Euclidean plane. The $n$ nodes $v_{0}, v_{1}, v_{2}, \ldots, v_{n-1}$ of $V$ are chosen randomly and independently from $U$ with a uniform distribution. For each node $v_{i}$, the $d$ nearest nodes in $V$ are made its neighbors, where $d \geq 1$. However, it is not guaranteed that $v_{i}$ and $v_{j}$ are in the set of $d$ nearest neighbors of each other. The actual neighbors are selected in the following way. First, we make an order of the nodes, say, $\left(v_{0}, v_{1}, v_{2}, \ldots, v_{n-1}\right)$. The degree of node $v_{i}$ is $d_{i}=0$ in the beginning. Then, for $0 \leq i \leq n-1$, assume that $v_{i}$ already had $d_{i}$ neighbors in $\left\{v_{0}, v_{1}, \ldots, v_{i-1}\right\}$. We choose the $d-d_{i}$ nearest neighbors of $v_{i}$ from the nodes in $\left\{v_{i+1}, v_{i+2}, \ldots, v_{n-1}\right\}$, say, $v_{j_{1}}, v_{j_{2}}$, ..., $v_{j_{d-d_{i}}}$, whose numbers of neighbors are still less $d$, and increase each of $d_{j_{1}}, d_{j_{2}}, \ldots, d_{j_{d-d_{i}}}$ by 1 .

A random unit disk network $N_{r}=(V, E)$ is generated as follows. The $n$ nodes $v_{0}, v_{1}, v_{2}, \ldots, v_{n-1}$ of $V$ are chosen randomly and independently from $U$ with a uniform distribution. Two nodes $v_{i}$ and $v_{j}$ are connected if and only if their distance is no longer than $r$, where $0 \leq r \leq 1 / 2$. The expected number of neighbors of a node is $n q_{r}$, where

$$
q_{r}=\pi r^{2}-\frac{8}{3} r^{3}+\left(\frac{11}{3}-\pi\right) r^{4},
$$

with $0 \leq r \leq 1 / 2$ [24].

Four cutsets are used for a random regular network and a random unit disk network (Figure 6), each cuts the unit square in a different way.

\subsection{Experimental Results}

All the sequences of random connection requests are generated by using the random drawing without replacement model. We believe that similar conclusions can be drawn by using the random drawing with replacement model.
We only consider connected random networks, that is, a random network is regenerated if it is disconnected. The parameters $q, d$, and $r$ of the three types of random networks are determined such that $q=0.9$ and $d=n q_{r}=10$. These parameter settings are to yield high connectedness of the random networks. To test the connectedness of the random networks with the above parameter settings, we generated 10,000 samples of each type of random networks. The numbers of connected samples of random grid networks, random regular networks, and random unit disk networks are $9213,9,999$, and 9,495, respectively.

Each experiment is repeated for 2000 times, and the 99\% confidence interval is shown for each table, which is obtained from the maximum confidence interval of all the experiments in a table. The $99 \%$ confidence interval is less than $\pm 2 \%$, except Table 8(a) for random unit disk networks. It is noticed that the number of wavelengths used on random unit disk networks has large variance. It has been observed that the probability distribution of the number of wavelengths used on random unit disk networks has a long tail, and the number of wavelengths may exceed, say, 256!

Our experimental data are displayed in Tables 1-8 for the eight WDM optical networks. Several observations are in order.

- All the four online algorithms exhibit excellent average-case performance on all the networks for the RWA problem, in the sense that for a wide range of $m$, both $\bar{\alpha}$ and $\bar{\beta}$ are very small (less than 2, except on random unit disk networks). In particular, as $m$ increases, both $\bar{\alpha}$ and $\bar{\beta}$ decrease and approach 1 . For the TM problem, high throughput can be achieved even for small $k$.

- The quality of $\bar{\alpha}$ and $\bar{\beta}$ depends on the quality of the lower bounds. We believe that the relatively large values of $\bar{\alpha}$ and $\bar{\beta}$ for the random unit disk networks are due to our inability to find tighter lower bounds. Those data in Table 8(a) obtained from loose lower bounds do not accurately reflect the average-case performance and certainly do not imply relatively poor performance of the four online algorithms on random unit disk networks.

- Though there is no dramatic difference among the performance of the four algorithms, Best-Fit is superior to all other algorithms in the sense that it yields smaller $\bar{\alpha}$ and $\bar{\beta}$, produces shorter average path length, and generates higher throughput.

- The average path length is quite stable and does not depend too much on the number of connection requests. 
Table 1(a). Experimental Data for RWA on the $10 \times 10$ Mesh Network. (99\% confidence interval $\pm 0.741 \%$ )

\begin{tabular}{|r|c|c|c|c|c|c|c|c|c|c|c|c|}
\hline \multirow{3}{*}{$m$} & \multicolumn{3}{|c|}{ First-Fit } & \multicolumn{3}{c|}{ Best-Fit } & \multicolumn{3}{c|}{ Densest-Fit } & \multicolumn{3}{|c|}{ Random-Fit } \\
\cline { 2 - 11 } & $\bar{\alpha}$ & $\bar{\beta}$ & $p l$ & $\bar{\alpha}$ & $\beta$ & $p l$ & $\bar{\alpha}$ & $\bar{\beta}$ & $\overline{p l}$ & $\bar{\alpha}$ & $\bar{\beta}$ & $\overline{p l}$ \\
\hline 50 & 1.622 & 1.738 & 7.512 & 1.633 & 1.742 & 7.111 & 1.741 & 1.861 & 7.378 & 1.677 & 1.800 & 7.448 \\
100 & 1.442 & 1.517 & 7.592 & 1.449 & 1.521 & 7.131 & 1.578 & 1.659 & 7.480 & 1.503 & 1.580 & 7.541 \\
150 & 1.372 & 1.428 & 7.633 & 1.368 & 1.428 & 7.153 & 1.518 & 1.583 & 7.525 & 1.432 & 1.492 & 7.576 \\
200 & 1.328 & 1.379 & 7.644 & 1.326 & 1.374 & 7.143 & 1.482 & 1.539 & 7.558 & 1.391 & 1.442 & 7.596 \\
250 & 1.302 & 1.348 & 7.657 & 1.292 & 1.339 & 7.147 & 1.456 & 1.508 & 7.570 & 1.365 & 1.407 & 7.610 \\
300 & 1.281 & 1.322 & 7.650 & 1.273 & 1.311 & 7.143 & 1.443 & 1.486 & 7.567 & 1.344 & 1.385 & 7.626 \\
350 & 1.266 & 1.302 & 7.653 & 1.257 & 1.292 & 7.155 & 1.431 & 1.471 & 7.585 & 1.330 & 1.366 & 7.624 \\
400 & 1.254 & 1.286 & 7.645 & 1.244 & 1.276 & 7.151 & 1.420 & 1.456 & 7.582 & 1.315 & 1.351 & 7.631 \\
450 & 1.243 & 1.274 & 7.652 & 1.231 & 1.262 & 7.156 & 1.411 & 1.446 & 7.596 & 1.306 & 1.336 & 7.634 \\
500 & 1.236 & 1.264 & 7.650 & 1.223 & 1.251 & 7.153 & 1.403 & 1.436 & 7.594 & 1.297 & 1.328 & 7.642 \\
\hline
\end{tabular}

Table 1(b). Experimental Data for TM on the $10 \times 10$ Mesh Network (99\% confidence interval $\pm 0.257 \%$ )

\begin{tabular}{|r|c|c|c|c|c|c|c|c|c|c|c|c|}
\hline & \multicolumn{3}{|c|}{ First-Fit } & \multicolumn{3}{c|}{ Best-Fit } & \multicolumn{3}{c|}{ Densest-Fit } & \multicolumn{3}{|c|}{ Random-Fit } \\
\cline { 2 - 11 }$m$ & 7 & 14 & 21 & 7 & 14 & 21 & 7 & 14 & 21 & 7 & 14 & 21 \\
\hline 50 & 1.000 & 1.000 & 1.000 & 1.000 & 1.000 & 1.000 & 1.000 & 1.000 & 1.000 & 1.000 & 1.000 & 1.000 \\
100 & 0.982 & 1.000 & 1.000 & 0.983 & 1.000 & 1.000 & 0.952 & 1.000 & 1.000 & 0.968 & 1.000 & 1.000 \\
150 & 0.769 & 1.000 & 1.000 & 0.784 & 1.000 & 1.000 & 0.756 & 1.000 & 1.000 & 0.760 & 1.000 & 1.000 \\
200 & 0.624 & 0.998 & 1.000 & 0.641 & 0.999 & 1.000 & 0.621 & 0.974 & 1.000 & 0.621 & 0.992 & 1.000 \\
250 & 0.529 & 0.906 & 1.000 & 0.543 & 0.918 & 1.000 & 0.529 & 0.874 & 1.000 & 0.529 & 0.887 & 1.000 \\
300 & 0.460 & 0.799 & 1.000 & 0.475 & 0.816 & 1.000 & 0.464 & 0.780 & 0.983 & 0.461 & 0.787 & 0.998 \\
350 & 0.410 & 0.714 & 0.964 & 0.424 & 0.733 & 0.972 & 0.414 & 0.706 & 0.919 & 0.411 & 0.707 & 0.941 \\
400 & 0.371 & 0.648 & 0.883 & 0.384 & 0.666 & 0.898 & 0.376 & 0.643 & 0.853 & 0.373 & 0.644 & 0.866 \\
450 & 0.341 & 0.594 & 0.813 & 0.352 & 0.612 & 0.832 & 0.344 & 0.593 & 0.793 & 0.343 & 0.592 & 0.799 \\
500 & 0.315 & 0.549 & 0.754 & 0.326 & 0.567 & 0.773 & 0.319 & 0.551 & 0.741 & 0.317 & 0.549 & 0.744 \\
\hline
\end{tabular}

Table 2(a). Experimental Data for RWA on a 24-node ARPANET Network. (99\% confidence interval $\pm 1.406 \%$ )

\begin{tabular}{|r|c|c|c|c|c|c|c|c|c|c|c|c|}
\hline \multirow{3}{*}{$m$} & \multicolumn{3}{|c|}{ First-Fit } & \multicolumn{3}{c|}{ Best-Fit } & \multicolumn{3}{c|}{ Densest-Fit } & \multicolumn{3}{c|}{ Random-Fit } \\
\cline { 2 - 11 } & $\bar{\alpha}$ & $\bar{\beta}$ & $\bar{l}$ & $\bar{\alpha}$ & $\beta$ & $\overline{p l}$ & $\bar{\alpha}$ & $\bar{\beta}$ & $\overline{p l}$ & $\bar{\alpha}$ & $\beta$ & $\overline{p l}$ \\
\hline 20 & 1.239 & 1.211 & 3.207 & 1.238 & 1.217 & 3.013 & 1.267 & 1.229 & 3.086 & 1.257 & 1.225 & 3.129 \\
40 & 1.120 & 1.098 & 3.244 & 1.114 & 1.099 & 2.984 & 1.136 & 1.123 & 3.109 & 1.124 & 1.107 & 3.173 \\
60 & 1.073 & 1.071 & 3.270 & 1.070 & 1.060 & 2.958 & 1.092 & 1.086 & 3.115 & 1.076 & 1.067 & 3.176 \\
80 & 1.051 & 1.049 & 3.265 & 1.047 & 1.048 & 2.945 & 1.068 & 1.065 & 3.115 & 1.055 & 1.053 & 3.189 \\
100 & 1.040 & 1.042 & 3.285 & 1.038 & 1.038 & 2.935 & 1.053 & 1.051 & 3.112 & 1.044 & 1.042 & 3.196 \\
120 & 1.032 & 1.027 & 3.277 & 1.032 & 1.033 & 2.920 & 1.044 & 1.046 & 3.108 & 1.035 & 1.038 & 3.197 \\
140 & 1.026 & 1.026 & 3.282 & 1.028 & 1.029 & 2.922 & 1.039 & 1.040 & 3.111 & 1.030 & 1.029 & 3.197 \\
160 & 1.023 & 1.020 & 3.281 & 1.023 & 1.020 & 2.908 & 1.034 & 1.035 & 3.107 & 1.025 & 1.028 & 3.203 \\
180 & 1.020 & 1.022 & 3.284 & 1.020 & 1.016 & 2.904 & 1.030 & 1.028 & 3.106 & 1.023 & 1.025 & 3.203 \\
200 & 1.018 & 1.014 & 3.282 & 1.019 & 1.019 & 2.906 & 1.027 & 1.028 & 3.108 & 1.020 & 1.020 & 3.199 \\
\hline
\end{tabular}

Table 2(b). Experimental Data for TM on a 24-node ARPANET Network. ( $99 \%$ confidence interval $\pm 0.435 \%$ )

\begin{tabular}{|r|c|c|c|c|c|c|c|c|c|c|c|c|}
\hline & \multicolumn{3}{|c|}{ First-Fit } & \multicolumn{3}{c|}{ Best-Fit } & \multicolumn{3}{c|}{ Densest-Fit } & \multicolumn{3}{|c|}{ Random-Fit } \\
\cline { 2 - 12 }$y$ & 3 & 6 & 9 & 3 & 6 & 9 & 3 & 6 & 9 & 3 & 6 & 9 \\
\hline 20 & 0.953 & 1.000 & 1.000 & 0.958 & 1.000 & 1.000 & 0.953 & 1.000 & 1.000 & 0.953 & 1.000 & 1.000 \\
40 & 0.793 & 0.974 & 1.000 & 0.800 & 0.972 & 1.000 & 0.781 & 0.972 & 1.000 & 0.783 & 0.973 & 1.000 \\
60 & 0.629 & 0.898 & 0.980 & 0.642 & 0.898 & 0.980 & 0.620 & 0.893 & 0.978 & 0.622 & 0.894 & 0.981 \\
80 & 0.515 & 0.829 & 0.927 & 0.530 & 0.834 & 0.929 & 0.514 & 0.809 & 0.925 & 0.515 & 0.814 & 0.927 \\
100 & 0.441 & 0.741 & 0.883 & 0.456 & 0.758 & 0.882 & 0.439 & 0.722 & 0.876 & 0.438 & 0.726 & 0.879 \\
120 & 0.385 & 0.661 & 0.843 & 0.400 & 0.682 & 0.846 & 0.385 & 0.647 & 0.821 & 0.385 & 0.650 & 0.827 \\
140 & 0.344 & 0.595 & 0.789 & 0.359 & 0.620 & 0.804 & 0.343 & 0.587 & 0.764 & 0.343 & 0.589 & 0.768 \\
160 & 0.312 & 0.544 & 0.729 & 0.325 & 0.570 & 0.753 & 0.311 & 0.539 & 0.709 & 0.312 & 0.539 & 0.712 \\
180 & 0.285 & 0.501 & 0.676 & 0.299 & 0.528 & 0.703 & 0.286 & 0.498 & 0.662 & 0.285 & 0.498 & 0.663 \\
200 & 0.263 & 0.465 & 0.631 & 0.276 & 0.491 & 0.658 & 0.264 & 0.464 & 0.619 & 0.264 & 0.464 & 0.621 \\
\hline
\end{tabular}

Table 3(a). Experimental Data for RWA on a 16-node NSFNET Backbone. (99\% confidence interval $\pm 1.437 \%)$

\begin{tabular}{|r|c|c|c|c|c|c|c|c|c|c|c|c|}
\hline \multirow{3}{*}{$m$} & \multicolumn{3}{|c|}{ First-Fit } & \multicolumn{3}{c|}{ Best-Fit } & \multicolumn{3}{c|}{ Densest-Fit } & \multicolumn{3}{|c|}{ Random-Fit } \\
\cline { 2 - 10 } & $\bar{\alpha}$ & $\beta$ & $p l$ & $\bar{\alpha}$ & $\beta$ & $p l$ & $\bar{\alpha}$ & $\beta$ & $p l$ & $\bar{\alpha}$ & $\beta$ & $p l$ \\
\hline 10 & 1.714 & 1.734 & 2.714 & 1.721 & 1.745 & 2.652 & 1.774 & 1.804 & 2.706 & 1.742 & 1.758 & 2.698 \\
20 & 1.498 & 1.471 & 2.736 & 1.512 & 1.487 & 2.653 & 1.572 & 1.557 & 2.743 & 1.531 & 1.521 & 2.754 \\
30 & 1.367 & 1.360 & 2.779 & 1.376 & 1.355 & 2.652 & 1.467 & 1.447 & 2.745 & 1.417 & 1.401 & 2.758 \\
40 & 1.303 & 1.294 & 2.770 & 1.300 & 1.288 & 2.661 & 1.396 & 1.387 & 2.762 & 1.343 & 1.338 & 2.772 \\
50 & 1.257 & 1.248 & 2.772 & 1.251 & 1.242 & 2.656 & 1.367 & 1.355 & 2.771 & 1.300 & 1.293 & 2.766 \\
60 & 1.221 & 1.218 & 2.779 & 1.218 & 1.215 & 2.659 & 1.334 & 1.329 & 2.767 & 1.268 & 1.262 & 2.776 \\
70 & 1.196 & 1.194 & 2.777 & 1.193 & 1.191 & 2.662 & 1.305 & 1.304 & 2.771 & 1.242 & 1.237 & 2.769 \\
80 & 1.177 & 1.173 & 2.778 & 1.175 & 1.173 & 2.663 & 1.288 & 1.284 & 2.770 & 1.222 & 1.218 & 2.777 \\
90 & 1.162 & 1.159 & 2.780 & 1.157 & 1.153 & 2.658 & 1.276 & 1.273 & 2.774 & 1.206 & 1.204 & 2.779 \\
100 & 1.149 & 1.146 & 2.779 & 1.145 & 1.142 & 2.663 & 1.263 & 1.259 & 2.770 & 1.190 & 1.189 & 2.778 \\
\hline
\end{tabular}

Table 3(b). Experimental Data for TM on a 16-node NSFNET Backbone. ( $99 \%$ confidence interval $\pm 0.549 \%$ )

\begin{tabular}{|r|c|c|c|c|c|c|c|c|c|c|c|c|}
\hline \multirow{3}{*}{$m$} & \multicolumn{3}{|c|}{ First-Fit } & \multicolumn{3}{c|}{ Best-Fit } & \multicolumn{3}{c|}{ Densest-Fit } & \multicolumn{3}{c|}{ Random-Fit } \\
\cline { 2 - 12 }$y$ & 3 & 6 & 9 & 3 & 6 & 9 & 3 & 6 & 9 & 3 & 6 & 9 \\
\hline 10 & 1.000 & 1.000 & 1.000 & 1.000 & 1.000 & 1.000 & 1.000 & 1.000 & 1.000 & 1.000 & 1.000 & 1.000 \\
20 & 0.900 & 1.000 & 1.000 & 0.900 & 1.000 & 1.000 & 0.879 & 1.000 & 1.000 & 0.887 & 1.000 & 1.000 \\
30 & 0.707 & 0.999 & 1.000 & 0.714 & 0.999 & 1.000 & 0.695 & 0.996 & 1.000 & 0.705 & 0.999 & 1.000 \\
40 & 0.585 & 0.950 & 1.000 & 0.595 & 0.952 & 1.000 & 0.581 & 0.920 & 1.000 & 0.582 & 0.935 & 1.000 \\
50 & 0.503 & 0.840 & 0.999 & 0.513 & 0.848 & 0.999 & 0.501 & 0.819 & 0.993 & 0.501 & 0.830 & 0.998 \\
60 & 0.445 & 0.754 & 0.974 & 0.454 & 0.762 & 0.974 & 0.445 & 0.739 & 0.940 & 0.444 & 0.744 & 0.958 \\
70 & 0.400 & 0.681 & 0.899 & 0.408 & 0.692 & 0.905 & 0.400 & 0.673 & 0.870 & 0.401 & 0.676 & 0.885 \\
80 & 0.366 & 0.625 & 0.833 & 0.373 & 0.637 & 0.839 & 0.365 & 0.619 & 0.810 & 0.365 & 0.622 & 0.818 \\
90 & 0.336 & 0.581 & 0.772 & 0.344 & 0.591 & 0.782 & 0.337 & 0.574 & 0.756 & 0.336 & 0.578 & 0.765 \\
100 & 0.313 & 0.542 & 0.724 & 0.321 & 0.555 & 0.734 & 0.313 & 0.538 & 0.712 & 0.314 & 0.539 & 0.717 \\
\hline
\end{tabular}

Table 4(a). Experimental Data for RWA on the 20-node European Optical Network. (99\% confidence interval $\pm 1.792 \%)$

\begin{tabular}{|r|c|c|c|c|c|c|c|c|c|c|c|c|}
\hline \multirow{3}{*}{$m$} & \multicolumn{3}{|c|}{ First-Fit } & \multicolumn{3}{c|}{ Best-Fit } & \multicolumn{3}{c|}{ Densest-Fit } & \multicolumn{3}{c|}{ Random-Fit } \\
\cline { 2 - 12 } & $\bar{\alpha}$ & $\bar{\beta}$ & $\overline{p l}$ & $\bar{\alpha}$ & $\bar{\beta}$ & $\overline{p l}$ & $\bar{\alpha}$ & $\bar{\beta}$ & $\overline{p l}$ & $\bar{\alpha}$ & $\bar{\beta}$ & $\overline{p l}$ \\
\hline 10 & 1.592 & 2.177 & 2.743 & 1.605 & 2.190 & 2.646 & 1.599 & 2.186 & 2.669 & 1.604 & 2.201 & 2.704 \\
20 & 1.409 & 1.728 & 2.803 & 1.408 & 1.719 & 2.646 & 1.418 & 1.742 & 2.726 & 1.399 & 1.718 & 2.765 \\
30 & 1.317 & 1.552 & 2.838 & 1.302 & 1.541 & 2.635 & 1.346 & 1.589 & 2.756 & 1.344 & 1.579 & 2.800 \\
40 & 1.264 & 1.446 & 2.849 & 1.253 & 1.443 & 2.641 & 1.305 & 1.489 & 2.783 & 1.300 & 1.475 & 2.808 \\
50 & 1.232 & 1.385 & 2.859 & 1.223 & 1.376 & 2.633 & 1.287 & 1.440 & 2.791 & 1.256 & 1.406 & 2.823 \\
60 & 1.211 & 1.333 & 2.868 & 1.203 & 1.325 & 2.625 & 1.266 & 1.387 & 2.806 & 1.236 & 1.361 & 2.831 \\
70 & 1.193 & 1.298 & 2.876 & 1.177 & 1.285 & 2.623 & 1.251 & 1.355 & 2.811 & 1.217 & 1.323 & 2.845 \\
80 & 1.178 & 1.263 & 2.873 & 1.163 & 1.258 & 2.618 & 1.232 & 1.326 & 2.822 & 1.197 & 1.285 & 2.850 \\
90 & 1.166 & 1.241 & 2.878 & 1.158 & 1.232 & 2.606 & 1.231 & 1.305 & 2.827 & 1.198 & 1.272 & 2.852 \\
100 & 1.150 & 1.211 & 2.882 & 1.150 & 1.214 & 2.603 & 1.220 & 1.286 & 2.835 & 1.181 & 1.244 & 2.858 \\
\hline
\end{tabular}

Table 4(b). Experimental Data for TM on the 20-node European Optical Network (99\% confidence interval $\pm 0.417 \%$ )

\begin{tabular}{|r|c|c|c|c|c|c|c|c|c|c|c|c|}
\hline \multirow{3}{*}{$m$} & \multicolumn{3}{|c|}{ First-Fit } & \multicolumn{3}{c|}{ Best-Fit } & \multicolumn{3}{c|}{ Densest-Fit } & \multicolumn{3}{|c|}{ Random-Fit } \\
\cline { 2 - 12 } & 3 & 6 & 9 & 3 & 6 & 9 & 3 & 6 & 9 & 3 & 6 & 9 \\
\hline 10 & 0.999 & 1.000 & 1.000 & 0.999 & 1.000 & 1.000 & 0.999 & 1.000 & 1.000 & 0.999 & 1.000 & 1.000 \\
20 & 0.982 & 1.000 & 1.000 & 0.981 & 1.000 & 1.000 & 0.980 & 1.000 & 1.000 & 0.980 & 1.000 & 1.000 \\
30 & 0.905 & 0.999 & 1.000 & 0.909 & 0.999 & 1.000 & 0.891 & 0.999 & 1.000 & 0.895 & 0.999 & 1.000 \\
40 & 0.780 & 0.996 & 1.000 & 0.789 & 0.996 & 1.000 & 0.768 & 0.996 & 1.000 & 0.770 & 0.996 & 1.000 \\
50 & 0.673 & 0.984 & 1.000 & 0.682 & 0.986 & 1.000 & 0.663 & 0.979 & 1.000 & 0.666 & 0.981 & 1.000 \\
60 & 0.587 & 0.952 & 0.999 & 0.601 & 0.953 & 0.999 & 0.586 & 0.930 & 0.999 & 0.585 & 0.941 & 0.999 \\
70 & 0.524 & 0.892 & 0.996 & 0.538 & 0.901 & 0.997 & 0.523 & 0.866 & 0.995 & 0.523 & 0.876 & 0.996 \\
80 & 0.473 & 0.822 & 0.989 & 0.488 & 0.839 & 0.989 & 0.475 & 0.801 & 0.980 & 0.474 & 0.809 & 0.986 \\
90 & 0.435 & 0.761 & 0.972 & 0.448 & 0.779 & 0.974 & 0.435 & 0.746 & 0.948 & 0.435 & 0.749 & 0.960 \\
100 & 0.402 & 0.705 & 0.937 & 0.416 & 0.725 & 0.942 & 0.403 & 0.695 & 0.906 & 0.402 & 0.699 & 0.919 \\
\hline
\end{tabular}


Table 5(a). Experimental Data for RWA on the 30-node UK Network. (99\% confidence interval $\pm 1.085 \%$ )

\begin{tabular}{|r|c|c|c|c|c|c|c|c|c|c|c|c|}
\hline \multirow{3}{*}{$m$} & \multicolumn{3}{|c|}{ First-Fit } & \multicolumn{3}{c|}{ Best-Fit } & \multicolumn{3}{c|}{ Densest-Fit } & \multicolumn{3}{|c|}{ Random-Fit } \\
\cline { 2 - 11 } & $\bar{\alpha}$ & $\beta$ & $p l$ & $\bar{\alpha}$ & $\bar{\beta}$ & $\overline{p l}$ & $\bar{\alpha}$ & $\bar{\beta}$ & $\overline{p l}$ & $\bar{\alpha}$ & $\bar{\beta}$ & $\overline{p l}$ \\
\hline 30 & 1.579 & 1.661 & 3.811 & 1.573 & 1.658 & 3.596 & 1.649 & 1.736 & 3.726 & 1.617 & 1.702 & 3.772 \\
60 & 1.400 & 1.431 & 3.845 & 1.401 & 1.436 & 3.617 & 1.506 & 1.541 & 3.780 & 1.438 & 1.481 & 3.826 \\
90 & 1.324 & 1.339 & 3.876 & 1.327 & 1.343 & 3.613 & 1.447 & 1.464 & 3.805 & 1.374 & 1.389 & 3.842 \\
120 & 1.277 & 1.289 & 3.889 & 1.284 & 1.291 & 3.616 & 1.413 & 1.420 & 3.822 & 1.329 & 1.337 & 3.856 \\
150 & 1.250 & 1.253 & 3.884 & 1.250 & 1.253 & 3.617 & 1.384 & 1.387 & 3.827 & 1.299 & 1.304 & 3.869 \\
180 & 1.229 & 1.229 & 3.889 & 1.225 & 1.226 & 3.618 & 1.364 & 1.367 & 3.840 & 1.277 & 1.279 & 3.875 \\
210 & 1.208 & 1.208 & 3.897 & 1.206 & 1.208 & 3.617 & 1.348 & 1.350 & 3.846 & 1.261 & 1.259 & 3.873 \\
240 & 1.195 & 1.194 & 3.900 & 1.191 & 1.192 & 3.617 & 1.336 & 1.336 & 3.848 & 1.248 & 1.245 & 3.880 \\
270 & 1.182 & 1.183 & 3.905 & 1.180 & 1.179 & 3.618 & 1.325 & 1.325 & 3.853 & 1.230 & 1.231 & 3.883 \\
300 & 1.171 & 1.171 & 3.905 & 1.166 & 1.166 & 3.614 & 1.317 & 1.315 & 3.850 & 1.219 & 1.220 & 3.884 \\
\hline
\end{tabular}

Table 5(b). Experimental Data for TM on the 30-node UK Network. (99\% confidence interval $\pm 0.339 \%)$

\begin{tabular}{|r|c|c|c|c|c|c|c|c|c|c|c|c|}
\hline \multirow{3}{*}{$m$} & \multicolumn{3}{|c|}{ First-Fit } & \multicolumn{3}{c|}{ Best-Fit } & \multicolumn{3}{c|}{ Densest-Fit } & \multicolumn{3}{c|}{ Random-Fit } \\
\cline { 2 - 12 } & 5 & 10 & 15 & 5 & 10 & 15 & 5 & 10 & 15 & 5 & 10 & 15 \\
\hline 30 & 1.000 & 1.000 & 1.000 & 1.000 & 1.000 & 1.000 & 1.000 & 1.000 & 1.000 & 1.000 & 1.000 & 1.000 \\
60 & 0.869 & 1.000 & 1.000 & 0.874 & 1.000 & 1.000 & 0.845 & 1.000 & 1.000 & 0.855 & 1.000 & 1.000 \\
90 & 0.662 & 0.997 & 1.000 & 0.676 & 0.997 & 1.000 & 0.657 & 0.986 & 1.000 & 0.658 & 0.995 & 1.000 \\
120 & 0.535 & 0.907 & 1.000 & 0.551 & 0.914 & 1.000 & 0.538 & 0.876 & 1.000 & 0.536 & 0.891 & 1.000 \\
150 & 0.455 & 0.782 & 0.994 & 0.470 & 0.800 & 0.994 & 0.458 & 0.770 & 0.968 & 0.457 & 0.774 & 0.986 \\
180 & 0.399 & 0.688 & 0.926 & 0.413 & 0.706 & 0.932 & 0.402 & 0.683 & 0.889 & 0.401 & 0.684 & 0.906 \\
210 & 0.356 & 0.616 & 0.839 & 0.369 & 0.635 & 0.852 & 0.361 & 0.616 & 0.815 & 0.359 & 0.614 & 0.824 \\
240 & 0.323 & 0.559 & 0.763 & 0.336 & 0.579 & 0.781 & 0.327 & 0.563 & 0.750 & 0.326 & 0.559 & 0.755 \\
270 & 0.297 & 0.513 & 0.702 & 0.309 & 0.533 & 0.721 & 0.302 & 0.519 & 0.696 & 0.299 & 0.515 & 0.697 \\
300 & 0.275 & 0.476 & 0.651 & 0.286 & 0.494 & 0.670 & 0.279 & 0.481 & 0.649 & 0.278 & 0.478 & 0.648 \\
\hline
\end{tabular}

Table 6(a). Experimental Data for RWA on 100-node Random Grid Networks. (99\% confidence interval $\pm 0.965 \%$ )

\begin{tabular}{|r|c|c|c|c|c|c|c|c|c|c|c|c|}
\hline \multirow{3}{*}{$m$} & \multicolumn{3}{|c|}{ First-Fit } & \multicolumn{3}{c|}{ Best-Fit } & \multicolumn{3}{c|}{ Densest-Fit } & \multicolumn{3}{|c|}{ Random-Fit } \\
\cline { 2 - 11 } & $\bar{\alpha}$ & $\bar{\beta}$ & $p l$ & $\bar{\alpha}$ & $\bar{\beta}$ & $\overline{p l}$ & $\bar{\alpha}$ & $\bar{\beta}$ & $\overline{p l}$ & $\bar{\alpha}$ & $\beta$ & $\bar{l}$ \\
\hline 50 & 1.875 & 2.025 & 8.093 & 1.889 & 2.048 & 7.603 & 1.998 & 2.164 & 7.958 & 1.926 & 2.082 & 8.006 \\
100 & 1.649 & 1.771 & 8.179 & 1.670 & 1.789 & 7.628 & 1.800 & 1.923 & 8.033 & 1.713 & 1.837 & 8.105 \\
150 & 1.567 & 1.673 & 8.191 & 1.582 & 1.687 & 7.638 & 1.718 & 1.831 & 8.066 & 1.621 & 1.727 & 8.110 \\
200 & 1.519 & 1.619 & 8.206 & 1.530 & 1.634 & 7.661 & 1.674 & 1.785 & 8.081 & 1.578 & 1.680 & 8.151 \\
250 & 1.493 & 1.584 & 8.206 & 1.495 & 1.600 & 7.666 & 1.636 & 1.736 & 8.099 & 1.538 & 1.636 & 8.154 \\
300 & 1.462 & 1.556 & 8.216 & 1.476 & 1.567 & 7.657 & 1.614 & 1.713 & 8.093 & 1.520 & 1.607 & 8.147 \\
350 & 1.446 & 1.533 & 8.214 & 1.457 & 1.546 & 7.670 & 1.599 & 1.698 & 8.098 & 1.495 & 1.594 & 8.174 \\
400 & 1.440 & 1.528 & 8.231 & 1.444 & 1.535 & 7.669 & 1.582 & 1.677 & 8.100 & 1.487 & 1.575 & 8.169 \\
450 & 1.424 & 1.503 & 8.220 & 1.440 & 1.523 & 7.681 & 1.573 & 1.670 & 8.111 & 1.476 & 1.559 & 8.176 \\
500 & 1.418 & 1.500 & 8.228 & 1.422 & 1.500 & 7.681 & 1.562 & 1.658 & 8.109 & 1.464 & 1.548 & 8.186 \\
\hline
\end{tabular}

Table 6(b). Experimental Data for TM on 100-node Random Grid Networks ( $99 \%$ confidence interval $\pm 0.554 \%$ )

\begin{tabular}{|r|c|c|c|c|c|c|c|c|c|c|c|c|}
\hline & \multicolumn{3}{|c|}{ First-Fit } & \multicolumn{3}{c|}{ Best-Fit } & \multicolumn{3}{c|}{ Densest-Fit } & \multicolumn{3}{|c|}{ Random-Fit } \\
\cline { 2 - 12 }$y$ & 7 & 14 & 21 & 7 & 14 & 21 & 7 & 14 & 21 & 7 & 14 & 21 \\
\hline 50 & 0.999 & 1.000 & 1.000 & 0.999 & 1.000 & 1.000 & 0.998 & 1.000 & 1.000 & 0.999 & 1.000 & 1.000 \\
100 & 0.791 & 1.000 & 1.000 & 0.803 & 1.000 & 1.000 & 0.783 & 1.000 & 1.000 & 0.787 & 1.000 & 1.000 \\
150 & 0.554 & 0.982 & 1.000 & 0.568 & 0.981 & 1.000 & 0.558 & 0.964 & 1.000 & 0.559 & 0.974 & 1.000 \\
200 & 0.433 & 0.832 & 0.997 & 0.442 & 0.847 & 0.997 & 0.437 & 0.822 & 0.993 & 0.437 & 0.827 & 0.995 \\
250 & 0.362 & 0.686 & 0.960 & 0.367 & 0.701 & 0.964 & 0.363 & 0.688 & 0.939 & 0.362 & 0.687 & 0.953 \\
300 & 0.312 & 0.586 & 0.857 & 0.315 & 0.597 & 0.866 & 0.312 & 0.589 & 0.844 & 0.313 & 0.587 & 0.845 \\
350 & 0.275 & 0.512 & 0.746 & 0.279 & 0.523 & 0.760 & 0.276 & 0.517 & 0.744 & 0.276 & 0.516 & 0.746 \\
400 & 0.248 & 0.458 & 0.662 & 0.250 & 0.467 & 0.679 & 0.247 & 0.461 & 0.666 & 0.247 & 0.461 & 0.663 \\
450 & 0.226 & 0.418 & 0.597 & 0.228 & 0.424 & 0.610 & 0.225 & 0.419 & 0.603 & 0.226 & 0.418 & 0.601 \\
500 & 0.208 & 0.383 & 0.547 & 0.209 & 0.389 & 0.559 & 0.207 & 0.384 & 0.550 & 0.208 & 0.383 & 0.550 \\
\hline
\end{tabular}

Table 7(a). Experimental Data for RWA on 50-node Random Regular Networks. (99\% confidence interval $\pm 1.706 \%)$

\begin{tabular}{|r|c|c|c|c|c|c|c|c|c|c|c|c|}
\hline & \multicolumn{3}{|c|}{ First-Fit } & \multicolumn{3}{c|}{ Best-Fit } & \multicolumn{3}{c|}{ Densest-Fit } & \multicolumn{3}{c|}{ Random-Fit } \\
\cline { 2 - 11 } & $\bar{\alpha}$ & $\beta$ & $p l$ & $\bar{\alpha}$ & $\beta$ & $p l$ & $\bar{\alpha}$ & $\beta$ & $p l$ & $\bar{\alpha}$ & $\beta$ & $p l$ \\
\hline 50 & 1.654 & 1.672 & 2.850 & 1.630 & 1.648 & 2.794 & 1.638 & 1.659 & 2.794 & 1.653 & 1.671 & 2.821 \\
100 & 1.669 & 1.894 & 2.973 & 1.677 & 1.911 & 2.811 & 1.728 & 1.965 & 2.849 & 1.687 & 1.929 & 2.919 \\
150 & 1.535 & 1.745 & 3.026 & 1.571 & 1.786 & 2.815 & 1.598 & 1.818 & 2.885 & 1.560 & 1.788 & 2.968 \\
200 & 1.487 & 1.671 & 3.055 & 1.504 & 1.706 & 2.827 & 1.551 & 1.776 & 2.898 & 1.512 & 1.714 & 2.993 \\
250 & 1.449 & 1.646 & 3.076 & 1.470 & 1.657 & 2.823 & 1.517 & 1.715 & 2.912 & 1.465 & 1.658 & 3.014 \\
300 & 1.423 & 1.615 & 3.092 & 1.438 & 1.631 & 2.831 & 1.487 & 1.697 & 2.924 & 1.443 & 1.647 & 3.022 \\
350 & 1.407 & 1.614 & 3.101 & 1.431 & 1.619 & 2.829 & 1.470 & 1.669 & 2.934 & 1.409 & 1.608 & 3.034 \\
400 & 1.396 & 1.575 & 3.105 & 1.404 & 1.589 & 2.828 & 1.461 & 1.653 & 2.934 & 1.405 & 1.610 & 3.044 \\
450 & 1.380 & 1.556 & 3.115 & 1.404 & 1.601 & 2.828 & 1.452 & 1.639 & 2.937 & 1.388 & 1.573 & 3.049 \\
500 & 1.358 & 1.544 & 3.120 & 1.391 & 1.584 & 2.824 & 1.434 & 1.632 & 2.942 & 1.380 & 1.559 & 3.059 \\
\hline
\end{tabular}

Table 7(b). Experimental Data for TM on 50-node Random Regular Networks. (99\% confidence interval $\pm 0.476 \%$ )

\begin{tabular}{|r|c|c|c|c|c|c|c|c|c|c|c|c|}
\hline & \multicolumn{3}{|c|}{ First-Fit } & \multicolumn{3}{c|}{ Best-Fit } & \multicolumn{3}{c|}{ Densest-Fit } & \multicolumn{3}{|c|}{ Random-Fit } \\
\cline { 2 - 12 } & 3 & 6 & 9 & 3 & 6 & 9 & 3 & 6 & 9 & 3 & 6 & 9 \\
\hline 50 & 1.000 & 1.000 & 1.000 & 1.000 & 1.000 & 1.000 & 1.000 & 1.000 & 1.000 & 1.000 & 1.000 & 1.000 \\
100 & 0.999 & 1.000 & 1.000 & 0.998 & 1.000 & 1.000 & 0.998 & 1.000 & 1.000 & 0.998 & 1.000 & 1.000 \\
150 & 0.967 & 1.000 & 1.000 & 0.966 & 1.000 & 1.000 & 0.960 & 1.000 & 1.000 & 0.964 & 1.000 & 1.000 \\
200 & 0.854 & 0.998 & 1.000 & 0.854 & 0.999 & 1.000 & 0.845 & 0.999 & 1.000 & 0.849 & 0.999 & 1.000 \\
250 & 0.738 & 0.994 & 1.000 & 0.745 & 0.992 & 1.000 & 0.737 & 0.991 & 0.999 & 0.738 & 0.993 & 1.000 \\
300 & 0.655 & 0.974 & 0.999 & 0.661 & 0.969 & 0.999 & 0.655 & 0.966 & 0.999 & 0.654 & 0.970 & 0.999 \\
350 & 0.589 & 0.925 & 0.997 & 0.599 & 0.925 & 0.995 & 0.591 & 0.914 & 0.995 & 0.590 & 0.922 & 0.996 \\
400 & 0.538 & 0.863 & 0.989 & 0.548 & 0.865 & 0.988 & 0.540 & 0.854 & 0.986 & 0.536 & 0.858 & 0.989 \\
450 & 0.496 & 0.799 & 0.973 & 0.508 & 0.806 & 0.972 & 0.498 & 0.796 & 0.968 & 0.494 & 0.799 & 0.974 \\
500 & 0.460 & 0.748 & 0.947 & 0.472 & 0.755 & 0.944 & 0.462 & 0.749 & 0.939 & 0.460 & 0.747 & 0.946 \\
\hline
\end{tabular}

Table 8(a). Experimental Data for RWA on 50-node Random Unit Disk Networks. (99\% confidence interval $\pm 5.123 \%$ )

\begin{tabular}{|r|c|c|c|c|c|c|c|c|c|c|c|c|}
\hline \multirow{3}{*}{$m$} & \multicolumn{3}{|c|}{ First-Fit } & \multicolumn{3}{c|}{ Best-Fit } & \multicolumn{3}{c|}{ Densest-Fit } & \multicolumn{3}{|c|}{ Random-Fit } \\
\cline { 2 - 11 } & $\bar{\alpha}$ & $\bar{\beta}$ & $p l$ & $\bar{\alpha}$ & $\bar{\beta}$ & $\overline{p l}$ & $\bar{\alpha}$ & $\beta$ & $p l$ & $\bar{\alpha}$ & $\bar{\beta}$ & $\overline{p l}$ \\
\hline 50 & 2.407 & 2.600 & 3.247 & 2.396 & 2.593 & 3.008 & 2.382 & 2.587 & 3.048 & 2.335 & 2.575 & 3.136 \\
100 & 2.443 & 2.887 & 3.370 & 2.515 & 3.033 & 3.000 & 2.518 & 2.977 & 3.105 & 2.489 & 2.918 & 3.195 \\
150 & 2.370 & 2.825 & 3.404 & 2.434 & 2.881 & 2.995 & 2.474 & 2.895 & 3.105 & 2.435 & 2.959 & 3.190 \\
200 & 2.357 & 2.732 & 3.436 & 2.374 & 2.827 & 2.995 & 2.345 & 2.768 & 3.125 & 2.357 & 2.780 & 3.216 \\
250 & 2.328 & 2.769 & 3.449 & 2.375 & 2.784 & 2.999 & 2.399 & 2.810 & 3.131 & 2.312 & 2.738 & 3.220 \\
300 & 2.293 & 2.744 & 3.455 & 2.376 & 2.850 & 2.980 & 2.328 & 2.745 & 3.143 & 2.389 & 2.786 & 3.231 \\
350 & 2.294 & 2.737 & 3.472 & 2.323 & 2.757 & 2.972 & 2.377 & 2.771 & 3.132 & 2.315 & 2.810 & 3.232 \\
400 & 2.266 & 2.714 & 3.466 & 2.274 & 2.705 & 2.966 & 2.336 & 2.783 & 3.131 & 2.253 & 2.684 & 3.237 \\
450 & 2.229 & 2.670 & 3.466 & 2.300 & 2.719 & 2.994 & 2.312 & 2.791 & 3.141 & 2.251 & 2.686 & 3.236 \\
500 & 2.291 & 2.697 & 3.482 & 2.234 & 2.685 & 2.967 & 2.308 & 2.699 & 3.134 & 2.206 & 2.670 & 3.228 \\
\hline
\end{tabular}

Table 8(b). Experimental Data for TM on 50-node Random Unit Disk Networks. ( $99 \%$ confidence interval $\pm 1.062 \%$ )

\begin{tabular}{|r|c|c|c|c|c|c|c|c|c|c|c|c|}
\hline \multirow{3}{*}{$m$} & \multicolumn{3}{|c|}{ First-Fit } & \multicolumn{3}{c|}{ Best-Fit } & \multicolumn{3}{c|}{ Densest-Fit } & \multicolumn{3}{c|}{ Random-Fit } \\
\cline { 2 - 12 } & 3 & 6 & 9 & 3 & 6 & 9 & 3 & 6 & 9 & 3 & 6 & 9 \\
\hline 50 & 0.981 & 0.997 & 0.999 & 0.979 & 0.996 & 0.998 & 0.981 & 0.997 & 0.999 & 0.978 & 0.996 & 0.999 \\
100 & 0.892 & 0.981 & 0.994 & 0.887 & 0.984 & 0.993 & 0.887 & 0.983 & 0.993 & 0.889 & 0.982 & 0.993 \\
150 & 0.763 & 0.950 & 0.984 & 0.768 & 0.948 & 0.983 & 0.767 & 0.947 & 0.983 & 0.762 & 0.947 & 0.983 \\
200 & 0.658 & 0.898 & 0.962 & 0.659 & 0.896 & 0.961 & 0.654 & 0.899 & 0.962 & 0.654 & 0.901 & 0.963 \\
250 & 0.570 & 0.833 & 0.937 & 0.575 & 0.836 & 0.930 & 0.567 & 0.834 & 0.936 & 0.573 & 0.840 & 0.933 \\
300 & 0.510 & 0.771 & 0.898 & 0.511 & 0.773 & 0.903 & 0.512 & 0.770 & 0.900 & 0.504 & 0.770 & 0.901 \\
350 & 0.457 & 0.712 & 0.859 & 0.464 & 0.712 & 0.862 & 0.458 & 0.715 & 0.856 & 0.458 & 0.717 & 0.857 \\
400 & 0.417 & 0.661 & 0.821 & 0.423 & 0.666 & 0.817 & 0.416 & 0.666 & 0.815 & 0.418 & 0.662 & 0.814 \\
450 & 0.382 & 0.619 & 0.772 & 0.389 & 0.621 & 0.780 & 0.382 & 0.615 & 0.774 & 0.383 & 0.615 & 0.781 \\
500 & 0.357 & 0.578 & 0.730 & 0.362 & 0.585 & 0.735 & 0.356 & 0.576 & 0.735 & 0.358 & 0.580 & 0.737 \\
\hline
\end{tabular}




\section{Concluding Remarks}

We have investigated the problem of online routing and wavelength assignment and the related throughput maximization problem in wavelength division multiplexing optical networks. It is very encouraging to find that even simple online RWA and TM algorithms can achieve excellent average-case competitive ratios. Our results also imply that the room for performance improvement by using offline algorithms is very limited.

\section{References}

[1] S. Appleby and S. Steward, "Mobile software agents for control in telecommunications networks," BT Technol. J., vol. 12, no. 2, pp. 104-113, 1994.

[2] B. Awerbuch, Y. Azar, and S. Plotkin, "Throughput-competitive online routing," Proceedings of the 34th Symposium on Foundations of Computer Science, pp. 32-40, 1993.

[3] B. Awerbuch, Y. Bartal, A. Fiat, and A. Rosén, "Competitive nonpreemptive call control," Proceedings of the 5th ACM-SIAM Symposium on Discrete Algorithms, pp. 312-320, 1994.

[4] B. Awerbuch, R. Gawlick, F. T. Leighton, and R. Rabani, "On-line admission control and circuit routing for high performance computing and communication," Proceedings of the 35th Symposium on Foundations of Computer Science, 1994.

[5] D. Banerjee and B. Mukherjee, "A practical approach for routing and wavelength assignment in large wavelength-routed optical networks," IEEE Journal on Selected Areas in Communications, vol. 14, no. 5, pp. 903-908, 1996.

[6] D. Banerjee and B. Mukherjee, "Wavelength-routed optical networks: linear formulation, resource budgeting tradeoffs, and a reconfiguration study," IEEE/ACM Transactions on Networking, vol. 8 , no. 5, pp. 598-607, 2000.

[7] Y. Bartal, A. Fiat, and S. Leonardi, "Lower bounds for on-line graph problems with application to on-line circuit and optical routing," Proceedings of the 28th ACM Symposium on Theory of Computing, pp. 531-540, 1996.

[8] Y. Bartal and S. Leonardi, "On-line routing in all-optical networks," Proceedings of the 24th International Colloquium on Automata, Languages and Programming, LNCS 1256, Springer-Verlag, 1997.

[9] D. Beckmann and U. Killat, "Routing and wavelength assignment in optical networks using genetic algorithms," European Transactions on Telecommunications, vol. 10, no. 5, pp. 537-544, 1999.

[10] A. Borodin and R. El-Yaniv, Online Computation and Competitive Analysis, Cambridge University Press, 1998.

[11] C. Chen and S. Banerjee, "A new model for optimal routing and wavelength assignment in wavelength division multiplexed optical network," Proceedings of IEEE Infocom, pp. 164-171, 1996.

[12] I. Chlamtac, A. Ganz, and G. Karmi, "Lightpath communications: an approach to high bandwidth optical WAN's," IEEE Transactions on Communications, vol. 40, no. 7, pp. 1171-1182, 1992.
[13] J. S. Choi, N. Golmie, F. Lapeyrere, F. Mouveaux, and D. Su, "A functional classification of routing and wavelength assignment schemes in DWDM networks: static case," Proceedings of the 7 th International Conference on Optical Communications and Networks, pp. 1109-1115, 2000.

[14] V. Guruswami, S. Khanna, R. Rajaraman, B. Shepherd, and M. Yannakakis, "Near-optimal hardness results and approximation algorithms for edge-disjoint paths and related problems," Proceedings of the 31st ACM Symposium on Theory of Computing, pp. 19-28, 1999.

[15] W. Feller, An Introduction to Probability Theory and Its Applications, Vol. I, 3rd edition, John Wiley \& Sons, New York, 1968.

[16] J. E. Freund, Statistics: A First Course, 3rd ed., Prentice-Hall, Englewood Cliffs, New Jersey, 1981.

[17] F. Harary, Graph Theory, Addison-Wesley, Reading, Massachusetts, 1969.

[18] S. Irani, "Coloring inductive graphs on-line," Proceedings of the 31 st Symposium on Foundations of Computer Science, pp. 470-479, 1990.

[19] H. A. Kierstead and W. T. Trotter, "An extremal problem in recursive combinatorics," Congressus Numerantium, vol. 33, pp. 143153, 1981.

[20] J. Kleinberg and E. Tardos, "Disjoint paths in densely embedded graphs," Proceedings of the 36st Symposium on Foundations of Computer Science, pp. 531-540, 1995.

[21] S. Leonardi, A. Marchetti-Spaccamela, A. Presciutti, and A. Rosén, "On-line randomized call control revisited," Proceedings of ACMSIAM Symposium on Discrete Algorithms, pp. 323-332, 1998.

[22] G. Li and R. Simha, "The partition coloring problem and its application to wavelength routing and assignment," Proceedings of Optical Networks Workshop, Richardson, Texas, 2000. See http://mozart.utdallas.edu/ONW2000/proceedings.

[23] K. Li, "Inapproximability results for wavelength assignment in wavelength division multiplexing optical networks," Proceedings of the 3rd International Conference on Communications in Computing, pp. 217-223, Las Vegas, Nevada, June 2002.

[24] K. Li, "Topological characteristics of random multihop wireless networks," Cluster Computing, vol. 8, pp. 119-126, 2005.

[25] M. J. O' Mahony, D. Simeonidou, A. Yu, and J. Zhou, "The design of a European optical network," Journal of Lightwave Technology, vol. 13 , no. 5 , pp. 817-828, 1995 .

[26] E. M. Palmer, Graphical Evolution, John Wiley \& Sons, New York, 1985.

[27] R. Ramaswami and K. N. Sivarajan, "Routing and wavelength assignment in all-optical networks," IEEE/ACM Transactions on Networking, vol. 3, no. 5, pp. 489-500, 1995.

[28] R. Ramaswami and K. N. Sivarajan, Optical Networks: A Practical Perspective, Morgan Kaufmann Publishers, San Francisco, California, 1998.

[29] Z. Zhang and A. S. Acampora, "A heuristic wavelength assignment algorithm for multihop WDM networks with wavelength routing and wavelength re-use," IEEE/ACM Transactions on Networking, vol. 3, no. 3, pp. 281-288, 1995. 